\title{
IS SEAWATER INTRUSION AFFECTING GROUND WATER ON LOPEZ ISLAND, WASHINGTON?
}

\section{HAS SEAWATER INTRUDED INTO LOPEZ ISLAND'S GROUND WATER?}

Lopez Island lies among the San Juan Islands, an archipelago in the coastal waters of Washington State, just offshore of Seattle and of Vancouver, British Columbia. Its scenic views and relatively little precipitation have made it one of Washington's premier places to live and play. So its population has been burgeoning, and its interior and shorelines have been under development.

The Island's main freshwater source is ground water. Local surface water cannot be developed to meet increasing needs for freshwater because the Island lacks lakes and continuously flowing streams. But Islanders are concerned that pumping more ground water will affect its availability and quality. Because many wells are near the shores and the recharge rates to the aquifers are low, there is a great potential for seawater intrusion.

In 1997, the U.S. Geological Survey (USGS), in cooperation with the San Juan County Conservation District, studied the possibilities of seawater intrusion on the Island and found that 46 percent of 185 freshwater samples had chloride concentrations indicating seawater intrusion.

\section{THE SOURCE OF LOPEZ ISLAND'S GROUND WATER}

Precipitation, mostly rain, is the main source of recharge to the Lopez Island's ground-water system. The Island, shielded by the rain shadow of the Olympic Mountains, receives 20 to 30 inches of precipitation a year, considerably less than other areas of western Washington more directly in the paths of storms from the Pacific Ocean (Oregon Climate Service, Oregon State University, 1999).

Some precipitation is lost to runoff and evapotranspiration. But some precipitation filters downward to recharge the groundwater system of aquifers made up geologically of unconsolidated glacial drift lying over a complex of sedimentary and volcanic bedrock that is metamorphosed in many areas. The glacial drift deposits of sand, gravel, silt, clay, and till cover an estimated 80 percent of the Island and vary in thickness from 0 to as great as 250 feet.

How much of the comparatively little recharge the Island gets each year depends on many factors - the distribution and intensity of the precipitation; the air temperature, and incident solar radiation; the amount and types of vegetation; the slope of the land; the

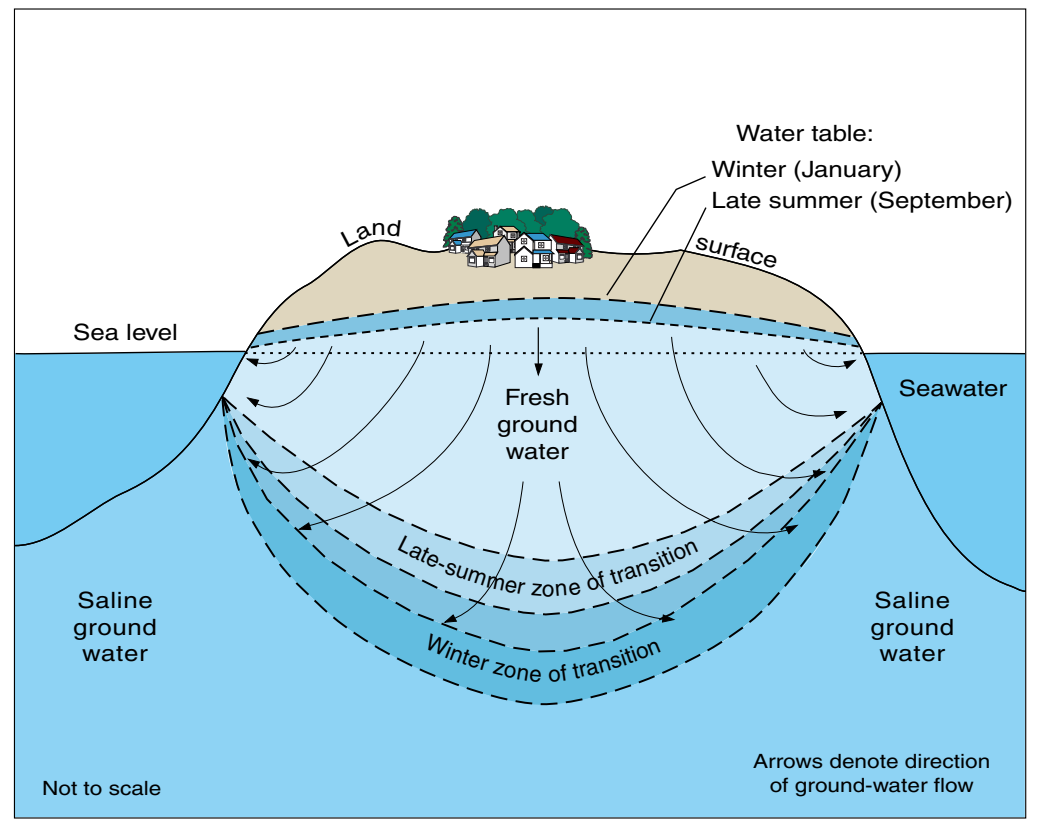

Figure 1. Generalized flow pattern of an homogeneous island aquifer. Movement of the zone of transition and water table shown for winter and late summer.

moisture-holding capacity of the soils; and the vertical permeability of the sediments above the aquifers.

But the small amount of yearly precipitation keeps the Island's ground-water system in a fragile balance between the recharge rates and the ground-water pumping. Increased pumping rates may upset this balance and result in seawater intrusion into nearshore aquifers.

\section{WHAT IS SEAWATER INTRUSION?}

In an unconfined aquifer that contacts the sea at the shoreline or seaward, the freshwater, which is less dense than seawater, floats as a lens-shaped layer on top of seawater (fig. 1), and the weight of the overlying freshwater depresses the seawater below sea level. Generally, freshwater recharge in these aquifers moves downgradient and eventually discharges to low-lying coastal areas and into the sea. But pumping out fresh ground water reduces the weight of the overlying freshwater, which in turn can decrease or even reverse the seaward flow so that seawater moves landward into the freshwater aquifer. This migration of seawater into the freshwater aquifer is known as seawater intrusion.

The interface between the salty ground water below and fresh ground water above is a transition zone (fig. 2) of gradually mixing fresh and salt waters. Under natural, undeveloped conditions, the location of this zone will move slightly as the tide rises or falls and as recharge fluctuates seasonally. However, when a well pumps fresh ground water from near the transition zone, the equilibrium can be disturbed and the groundwater flow pattern changed (fig. 2b). As water is pumped out of the water-bearing zone, the transition zone moves upward toward the well. Prolonged or large-scale pumping can raise the transition zone to the well, which may then draw in salty water (fig. 2c).

Withdrawing freshwater from a well affects not only the location of the transition zone around that well but also the location of the Island's regional transition zone. Thus, pumping wells, whether shallow or deep, no matter what their locations, will affect the whole Island's fresh-water system (fig. 1).

The location of the transition zone depends on several natural and human-made conditions: the relative densities of seawater and freshwater; the tides; the pumpage from 
wells; the rate of ground-water recharge; and the hydraulic characteristics of the aquifer. Because these conditions vary locally, the depth to the transition zone below sea level differs from one place to another on the Island.

\section{WHAT INDICATES SEAWATER INTRUSION?}

One indicator of seawater intrusion is an increased chloride concentration in a freshwater aquifer, because chloride, a major constituent of seawater, is chemically stable and moves at about the same rate as intruding seawater. For the purposes of this study, chloride concentrations of 100 milligrams per liter $(\mathrm{mg} / \mathrm{L})$ or more were assumed to indicate seawater intrusion.

This study's indication of seawater was also used in a previous USGS study conducted in San Juan County in 1981. That 1981 study used graphical analysis and the cumulative frequency distribution of chloride concentrations to establish a threshold value of $100 \mathrm{mg} / \mathrm{L}$ for seawater intrusion (Whiteman and others, 1983).

Seawater contains approximately $35,000 \mathrm{mg} / \mathrm{L}$ of dissolved solids, which include about $19,000 \mathrm{mg} / \mathrm{L}$ of chloride. Fresh ground water in most coastal areas of Washington generally contains less than 10 $\mathrm{mg} / \mathrm{L}$ of chloride. Even so, concentrations in excess of $10 \mathrm{mg} / \mathrm{L}$ are not conclusive evidence of seawater intrusion because they could be due to airborne sea spray in precipitation, to substantial well pumping rates, to local sources of chlorides, including septic systems or animal manure, or to relict seawater in the aquifer.

At times during the last million and a half years, the sea level along the Washington coastline was higher than now, and the transition zone between fresh and salty ground water was correspondingly farther inland and at higher elevations. Today, occurrences of salt water in Washington coastal aquifers may be due to relict seawater - seawater incompletely flushed from rock materials after the latest decline of sea level. The term relict seawater can also refer to connate water, or water trapped in an aquifer since its formation (Dion and Sumioka, 1984).

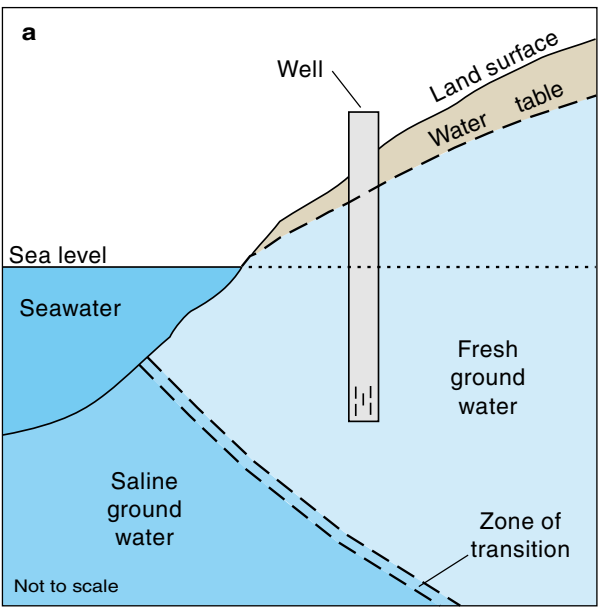

Nonpumping well in an unconfined (water-table) aquife under conditions of equilibrium--no intrusion has occurred.

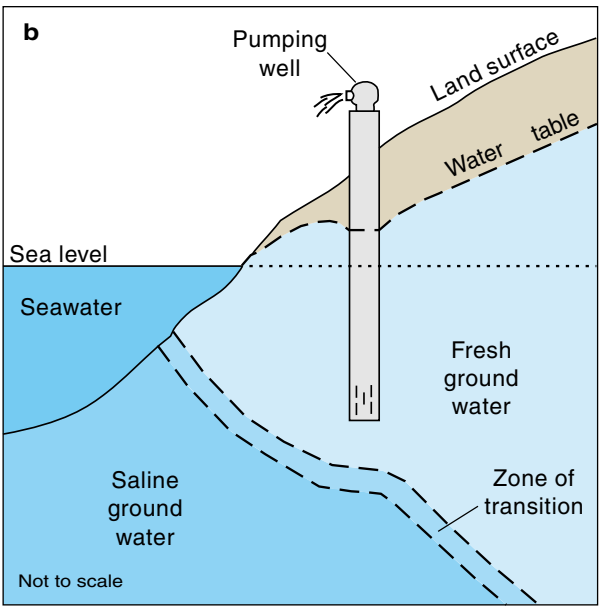

Well pumping from an unconfined (water-table) aquifer-seawater intrusion not affecting salinity of pumped water

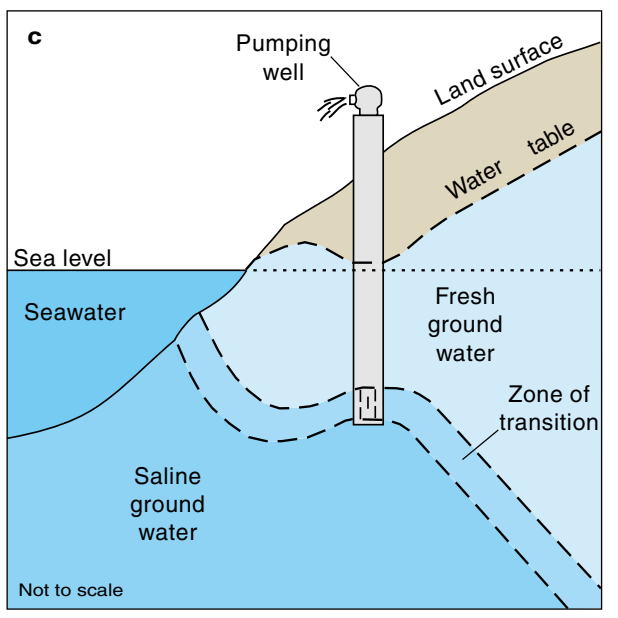

Well pumping from an unconfined aquifer--seawater intrusion affecting salinity of pumped water.

Figure 2. Hypothetical hydrologic conditions before and after seawater intrusion.
WHAT CAN HELP REDUCE SEAWATER INTRUSION?

Seawater intrusion on Lopez Island can be minimized by water conservation, efficient well construction, and by judicious well-operation practices like these:

- Using such water-conserving devices as low-volume plumbing fixtures and toilets.

- Keeping outdoor watering to a minimum.

- Reusing or recycling water when possible.

- Augmenting fresh groundwater recharge by, for example, using surface ponds to slow surface runoff and raise infiltration rates.

- Constructing wells that do not penetrate deeper below sea level than necessary.

- Sizing pumps for lower pumping rates and minimizing lengths of pumping cycles.

- In multiple-well systems, pumping wells alternately. 


\section{1-to-40 and the GHYBEN-HERZBERG PRINCIPLE}

In general, if the water table in an aquifer is lowered $1 \mathrm{foot}$, the freshwater-seawater transition zone will rise about 40 feet, and the total vertical thickness of the freshwater lens will be reduced by about 41 feet (Freeze and Cherry, 1979).

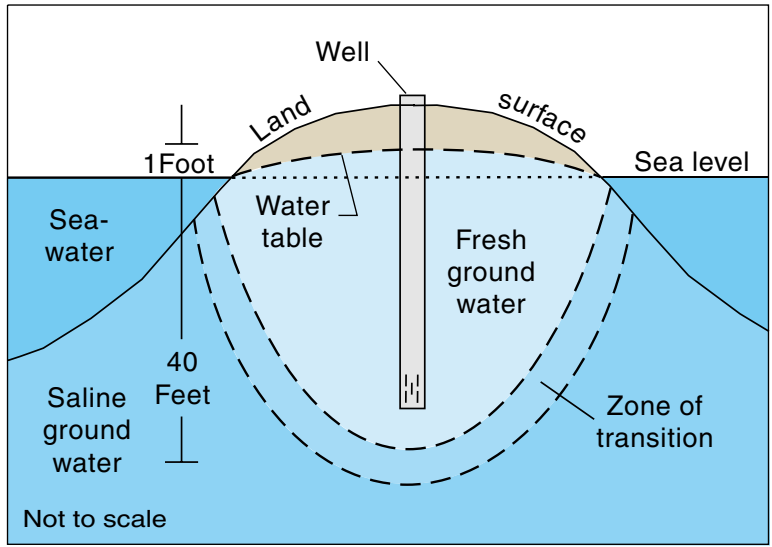

A century ago, hydrologists working along Europe's coast observed that fresh ground water, appearing to float as a lens-shaped body on seawater, extended below sea level approximately 40 times the height of the freshwater table above sea level. Named the Ghyben-Herzberg Principle after the two scientists who described it, this 1-to-40 relation occurs because freshwater is slightly less dense than seawater $(1.000$ grams per cubic centimeter $\left(\mathrm{g} / \mathrm{cm}^{3}\right)$ versus 1.025 $\mathrm{g} / \mathrm{cm}^{3}$ ). Thus, for example, if the water table at a given site is 3 feet above sea level, the freshwaterseawater transition zone is 120 feet below sea level, and the vertical thickness of the freshwater body there is 123 feet.

\section{SAMPLING, ANALYSIS, AND QUALITY ASSURANCE}

In spring 1997, after USGS scientists had reviewed data from more than 400 possible sites, giving priority to those previously visited (Whiteman and others, 1983 and Dion and Sumioka, 1984), field personnel visited 258 wells and a spring (see table 1) representing the Island.

Water samples from 184 wells and one spring (fig. 3 and table 1) were collected for analysis of specific conductance and chloride concentration. Specific conductance measurements were determined at the USGS Tacoma Field Service Unit (Tacoma FSU), Tacoma, Wash. The chloride content was determined colorimetrically using ferric thiocyanate (Friedman and Erdmann, 1982) at the Tacoma FSU.

Replicate and blank samples were collected and analyzed for chloride at the Tacoma FSU and at the USGS Quality of Water Service Unit in Ocala, Fla., in accordance with the Quality Assurance Plan for Water-Quality Activities of the Pacific Northwest District (Bortleson, U.S. Geological Survey, written commun., 1991). For every six samples, one sample of deionized water blanks was collected and analyzed at the Tacoma FSU. The results for all samples were acceptable. All replicates analyzed at both Tacoma and Ocala agreed within 5 percent of the replicate mean. Reference samples were within 5 percent of the known concentration of chloride. Chloride was not detected in any of the blank samples. The resulting field and quality-assurance data were reviewed and stored in the National Water Information System (Garcia and others, 1997).

\section{WHAT THE STUDY FOUND}

The 1997 study found chloride concentrations of $100 \mathrm{mg} / \mathrm{L}$ or more in 46 percent of the Island's 185 sites, indicating possible seawater intrusion. Chloride concentrations from the 185 ground-water samples ranged from $12 \mathrm{mg} / \mathrm{L}$ to $420 \mathrm{mg} / \mathrm{L}$, with a median value of $92 \mathrm{mg} / \mathrm{L}$.

When the 1997 and the 1981 chloride data were compared, there was no evident change in the areal distribution of chloride values from 1981 to 1997. Both chloride data sets had similar patterns of lowest concentrations near the center of the Island and highest concentrations mainly near the Island's southwestern, western, and northern shores. Moreover, there was no distinct pattern of changes of chloride concentrations in individual wells.

Of wells completed in bedrock units, 56 percent ( 28 wells) evinced seawater intrusion, while 39 percent of wells completed in glacial drift units (42 sites) showed such signs (fig. 1). One reason the bedrock wells may have shown more seawater intrusion was because they are generally deeper and thus closer to the transition zone. 
Table 1. Summary of concentrations of chlorides, physical data, and hydrologic data for wells and a spring sampled in 1997 on Lopez Island [Hydrogeologic unit: B, basalt; G, glacial; M, gravel and basalt; mg/L as CL, milligrams per liter of Chloride; —, no data; . Chloride data rounded to two significant digits, all other data rounded to nearest foot; Altitude of land surface is based on sea level (NGVD of 1927)]

\begin{tabular}{|c|c|c|c|c|c|c|c|c|}
\hline \multirow{2}{*}{$\begin{array}{l}\text { Station name (town- } \\
\text { ship/range-section and } \\
\text { sequence number) }\end{array}$} & \multirow{2}{*}{$\begin{array}{l}\text { Altitude of land } \\
\text { surface (feet) }\end{array}$} & \multirow{2}{*}{$\begin{array}{l}\text { Well depth } \\
\text { (feet below } \\
\text { land surface) }\end{array}$} & \multirow{2}{*}{$\begin{array}{l}\text { Depth to first } \\
\text { opening of } \\
\text { well (feet) }\end{array}$} & \multirow{2}{*}{$\begin{array}{c}\text { Spring } 1997 \\
\text { water-level } \\
\text { altitude (feet) }\end{array}$} & \multicolumn{3}{|c|}{$\begin{array}{l}\text { Chloride dissolved } \\
\text { (mg/L as } \mathrm{CL} \text { ) }\end{array}$} & \multirow{2}{*}{$\begin{array}{c}\text { Hydrogeologic } \\
\text { unit }\end{array}$} \\
\hline & & & & & $\begin{array}{l}\text { Spring } \\
1997\end{array}$ & $\begin{array}{c}\text { Spring } \\
1981\end{array}$ & $\begin{array}{l}\text { Spring } \\
1978\end{array}$ & \\
\hline 34N/1W-05H1 & 40 & 340 & 132 & 41 & 190 & - & - & $\mathrm{B}$ \\
\hline 34N/1W-05R1 & 90 & 400 & 59 & - & 120 & - & - & G \\
\hline 34N/1W-06B1 & 100 & 270 & 35 & - & 120 & 85 & - & B \\
\hline $34 \mathrm{~N} / 1 \mathrm{~W}-06 \mathrm{C} 2$ & 200 & 200 & - & 188 & 62 & - & - & - \\
\hline $34 \mathrm{~N} / 1 \mathrm{~W}-06 \mathrm{C} 3$ & 140 & 315 & 25 & - & 82 & - & - & B \\
\hline 34N/1W-06L1 & 40 & 214 & 41 & 10 & 30 & 34 & 28 & $\mathrm{~B}$ \\
\hline $34 \mathrm{~N} / 1 \mathrm{~W}-07 \mathrm{G} 2$ & 170 & 184 & 184 & - & 190 & - & - & $\mathrm{G}$ \\
\hline 34N/1W-07H1 & 135 & 174 & 147 & - & 260 & 170 & - & B \\
\hline 34N/1W-07Q1 & 40 & 270 & - & - & 290 & 150 & 150 & - \\
\hline 34N/1W-09M1 & 130 & 168 & 163 & 27 & 76 & - & - & $\mathrm{G}$ \\
\hline 34N/1W-09P1 & 105 & 440 & 232 & - & 270 & - & - & B \\
\hline 34N/1W-09R1 & 60 & 164 & 30 & 34 & 87 & 87 & 72 & B \\
\hline $34 \mathrm{~N} / 1 \mathrm{~W}-16 \mathrm{~B} 1$ & 75 & 143 & 138 & 72 & 150 & 130 & 110 & $\mathrm{~B}$ \\
\hline 34N/1W-16D1 & 150 & 112 & 112 & - & 72 & - & - & $\mathrm{G}$ \\
\hline $34 \mathrm{~N} / 1 \mathrm{~W}-16 \mathrm{D} 3$ & 165 & 300 & - & - & 110 & - & - & - \\
\hline $34 \mathrm{~N} / 1 \mathrm{~W}-16 \mathrm{G} 1$ & 90 & 338 & 44 & 66 & 90 & - & - & $\mathrm{B}$ \\
\hline 34N/1W-17A1 & 180 & 198 & 192 & - & 96 & - & - & $\mathrm{G}$ \\
\hline $34 \mathrm{~N} / 1 \mathrm{~W}-17 \mathrm{~B} 2$ & 180 & 299 & 262 & 19 & 130 & - & - & M \\
\hline 34N/1W-17D1 & 200 & 250 & 32 & - & 170 & 100 & - & $\mathrm{B}$ \\
\hline 34N/1W-17D2 & 220 & 152 & 18 & 205 & 150 & - & - & $\mathrm{G}$ \\
\hline 34N/1W-17E1 & 70 & 115 & 106 & 13 & 56 & 59 & 52 & $\mathrm{G}$ \\
\hline $34 \mathrm{~N} / 1 \mathrm{~W}-17 \mathrm{E} 2$ & 70 & 87 & 82 & 17 & 90 & - & - & G \\
\hline 34N/1W-17G1 & 150 & 290 & 40 & - & 200 & - & - & B \\
\hline $34 \mathrm{~N} / 1 \mathrm{~W}-17 \mathrm{G} 2$ & 170 & 124 & 19 & - & 70 & - & - & B \\
\hline $34 \mathrm{~N} / 1 \mathrm{~W}-17 \mathrm{~N} 2$ & 150 & 142 & - & - & 200 & - & - & - \\
\hline 34N/1W-17P1 & 110 & 170 & 99 & 45 & 170 & 100 & - & B \\
\hline $34 \mathrm{~N} / 1 \mathrm{~W}-18 \mathrm{C} 1$ & 140 & 259 & 102 & - & 260 & - & - & B \\
\hline 34N/1W-18E2 & 120 & 260 & 75 & - & 280 & 350 & 410 & B \\
\hline $34 \mathrm{~N} / 1 \mathrm{~W}-18 \mathrm{~F} 2$ & 100 & 35 & - & 97 & 260 & - & - & - \\
\hline 34N/1W-18G1D1 & 80 & 132 & 132 & 6 & 160 & 150 & - & $\mathrm{G}$ \\
\hline 34N/1W-18H1 & 100 & 115 & 115 & - & 82 & 46 & - & $\mathrm{G}$ \\
\hline $34 \mathrm{~N} / 1 \mathrm{~W}-18 \mathrm{~K} 1$ & 110 & 15 & - & 108 & 48 & - & - & - \\
\hline $34 \mathrm{~N} / 1 \mathrm{~W}-18 \mathrm{~L} 2$ & 15 & 46 & 41 & - & 80 & - & - & $\mathrm{G}$ \\
\hline 34N/1W-18L3 & 20 & 104 & 99 & - & 260 & - & - & $\mathrm{G}$ \\
\hline $34 \mathrm{~N} / 1 \mathrm{~W}-18 \mathrm{~N} 1$ & 20 & 62 & 62 & - & 23 & - & - & G \\
\hline $34 \mathrm{~N} / 1 \mathrm{~W}-18 \mathrm{P} 1$ & 40 & 58 & 50 & 27 & 280 & - & - & G \\
\hline $34 \mathrm{~N} / 1 \mathrm{~W}-18 \mathrm{P} 2$ & 60 & 52 & 47 & 43 & 94 & - & - & G \\
\hline 34N/1W-19N1 & 70 & 69 & 68 & 48 & 180 & - & - & $\mathrm{G}$ \\
\hline $34 \mathrm{~N} / 1 \mathrm{~W}-19 \mathrm{~N} 1 \mathrm{~S}$ & 40 & spring & - & - & 270 & - & - & - \\
\hline $34 \mathrm{~N} / 1 \mathrm{~W}-20 \mathrm{E} 1$ & 30 & 164 & 11 & 18 & 52 & 73 & 42 & $\mathrm{G}$ \\
\hline 34N/1W-21E1 & 80 & 30 & - & 77 & 110 & - & - & - \\
\hline $34 \mathrm{~N} / 1 \mathrm{~W}-21 \mathrm{H} 2$ & 70 & 345 & 20 & 38 & 400 & - & - & $\mathrm{B}$ \\
\hline $34 \mathrm{~N} / 1 \mathrm{~W}-21 \mathrm{H} 3$ & 55 & - & - & - & 64 & - & - & - \\
\hline $34 \mathrm{~N} / 1 \mathrm{~W}-21 \mathrm{M} 1$ & 80 & 200 & - & 44 & 260 & - & - & - \\
\hline $34 \mathrm{~N} / 1 \mathrm{~W}-21 \mathrm{M} 2$ & 100 & 500 & - & 21 & 250 & - & - & - \\
\hline 34N/2W-01M1 & 260 & 260 & - & - & 100 & - & - & - \\
\hline $34 \mathrm{~N} / 2 \mathrm{~W}-02 \mathrm{~B} 1$ & 270 & 266 & 20 & 251 & 42 & 30 & - & B \\
\hline $34 \mathrm{~N} / 2 \mathrm{~W}-02 \mathrm{D} 1$ & 155 & 307 & 65 & 130 & 140 & - & - & $\mathrm{B}$ \\
\hline $34 \mathrm{~N} / 2 \mathrm{~W}-02 \mathrm{E} 1$ & 170 & 250 & 160 & - & 94 & - & - & B \\
\hline 34N/2W-02J1 & 225 & 300 & 19 & - & 170 & - & - & B \\
\hline $34 \mathrm{~N} / 2 \mathrm{~W}-02 \mathrm{~J} 2$ & 205 & 30 & - & 203 & 12 & - & - & - \\
\hline $34 \mathrm{~N} / 2 \mathrm{~W}-02 \mathrm{P} 1$ & 140 & 414 & 23 & - & 230 & 180 & 150 & B \\
\hline $34 \mathrm{~N} / 2 \mathrm{~W}-03 \mathrm{~A} 1$ & 150 & 226 & 226 & - & 70 & - & - & $\mathrm{G}$ \\
\hline $34 \mathrm{~N} / 2 \mathrm{~W}-03 \mathrm{~B} 1$ & 180 & 194 & 194 & 1 & 88 & - & - & $\mathrm{G}$ \\
\hline $34 \mathrm{~N} / 2 \mathrm{~W}-03 \mathrm{C} 1$ & 200 & 229 & 199 & - & 86 & 67 & - & $\mathrm{G}$ \\
\hline $34 \mathrm{~N} / 2 \mathrm{~W}-03 \mathrm{D} 1$ & 180 & 216 & 216 & - & 78 & - & - & G \\
\hline $34 \mathrm{~N} / 2 \mathrm{~W}-03 \mathrm{~F} 1$ & 130 & 154 & 144 & - & 150 & 120 & - & G \\
\hline $34 \mathrm{~N} / 2 \mathrm{~W}-03 \mathrm{H} 1$ & 170 & 204 & 204 & 7 & 86 & - & - & G \\
\hline $34 \mathrm{~N} / 2 \mathrm{~W}-03 \mathrm{~J} 1$ & 170 & 181 & 176 & - & 100 & - & - & $\mathrm{G}$ \\
\hline $34 \mathrm{~N} / 2 \mathrm{~W}-03 \mathrm{~L} 1$ & 145 & 265 & 135 & - & 140 & 92 & - & $\mathrm{G}$ \\
\hline $34 \mathrm{~N} / 2 \mathrm{~W}-03 \mathrm{~N} 1$ & 105 & 166 & 166 & 35 & 110 & - & - & B \\
\hline
\end{tabular}


Table 1. Summary of concentrations of chlorides, physical data, and hydrologic data for wells and a spring sampled in 1997 on Lopez Island-Continued

\begin{tabular}{|c|c|c|c|c|c|c|c|c|}
\hline \multirow{2}{*}{$\begin{array}{l}\text { Station name (town- } \\
\text { ship/range-section and } \\
\text { sequence number) }\end{array}$} & \multirow{2}{*}{$\begin{array}{l}\text { Altitude of land } \\
\text { surface (feet) }\end{array}$} & \multirow{2}{*}{$\begin{array}{l}\text { Well depth } \\
\text { (feet below } \\
\text { land surface) }\end{array}$} & \multirow{2}{*}{$\begin{array}{l}\text { Depth to first } \\
\text { opening of } \\
\text { well (feet) }\end{array}$} & \multirow{2}{*}{$\begin{array}{c}\text { Spring } 1997 \\
\text { water-level } \\
\text { altitude (feet) }\end{array}$} & \multicolumn{3}{|c|}{$\begin{array}{l}\text { Chloride dissolved } \\
\text { (mg/L as CL) }\end{array}$} & \multirow{2}{*}{$\begin{array}{c}\text { Hydrogeologic } \\
\text { unit }\end{array}$} \\
\hline & & & & & $\begin{array}{l}\text { Spring } \\
1997\end{array}$ & $\begin{array}{c}\text { Spring } \\
1981\end{array}$ & $\begin{array}{l}\text { Spring } \\
1978\end{array}$ & \\
\hline $34 \mathrm{~N} / 2 \mathrm{~W}-04 \mathrm{~B} 2$ & 82 & 96 & 96 & - & 92 & - & - & G \\
\hline $34 \mathrm{~N} / 2 \mathrm{~W}-04 \mathrm{~B} 4$ & 85 & 90 & 90 & 10 & 88 & - & - & G \\
\hline $34 \mathrm{~N} / 2 \mathrm{~W}-04 \mathrm{G} 2$ & 80 & 88 & 88 & 11 & 100 & - & - & G \\
\hline $34 \mathrm{~N} / 2 \mathrm{~W}-04 \mathrm{H} 2$ & 150 & 169 & 169 & 3 & 100 & - & - & $\mathrm{G}$ \\
\hline $34 \mathrm{~N} / 2 \mathrm{~W}-04 \mathrm{~K} 1$ & 80 & 240 & 52 & 8 & 94 & - & - & B \\
\hline $34 \mathrm{~N} / 2 \mathrm{~W}-09 \mathrm{~A} 1$ & 62 & 134 & 129 & - & 210 & - & - & G \\
\hline $34 \mathrm{~N} / 2 \mathrm{~W}-10 \mathrm{~B} 1$ & 65 & 16 & - & - & 94 & - & - & - \\
\hline $34 \mathrm{~N} / 2 \mathrm{~W}-10 \mathrm{C} 2$ & 90 & 244 & 19 & - & 92 & - & - & B \\
\hline $34 \mathrm{~N} / 2 \mathrm{~W}-10 \mathrm{D} 1$ & 20 & 52 & 46 & -4 & 54 & - & - & G \\
\hline $34 \mathrm{~N} / 2 \mathrm{~W}-10 \mathrm{R} 3$ & 50 & 330 & 28 & - & 230 & - & - & M \\
\hline $34 \mathrm{~N} / 2 \mathrm{~W}-11 \mathrm{~A} 1$ & 125 & 106 & 106 & 87 & 150 & - & - & B \\
\hline $34 \mathrm{~N} / 2 \mathrm{~W}-11 \mathrm{~F} 1$ & 70 & 272 & 46 & 55 & 110 & - & - & B \\
\hline $34 \mathrm{~N} / 2 \mathrm{~W}-11 \mathrm{~N} 4$ & 20 & 150 & 39 & - & 320 & - & - & M \\
\hline $34 \mathrm{~N} / 2 \mathrm{~W}-12 \mathrm{~A} 2$ & 150 & 40 & - & 148 & 58 & - & - & - \\
\hline $34 \mathrm{~N} / 2 \mathrm{~W}-12 \mathrm{D} 1$ & 130 & 134 & 25 & 103 & 130 & 47 & - & B \\
\hline $34 \mathrm{~N} / 2 \mathrm{~W}-12 \mathrm{E} 1$ & 100 & 179 & 18 & - & 400 & - & - & B \\
\hline $34 \mathrm{~N} / 2 \mathrm{~W}-12 \mathrm{G} 1$ & 150 & 196 & 20 & 132 & 100 & 73 & - & B \\
\hline $34 \mathrm{~N} / 2 \mathrm{~W}-12 \mathrm{M} 1$ & 110 & 252 & 18 & - & 280 & - & - & B \\
\hline $34 \mathrm{~N} / 2 \mathrm{~W}-12 \mathrm{~N} 1$ & 70 & 328 & 20 & - & 120 & - & - & B \\
\hline $34 \mathrm{~N} / 2 \mathrm{~W}-12 \mathrm{P} 1$ & 135 & 265 & 42 & - & 86 & 180 & - & B \\
\hline $34 \mathrm{~N} / 2 \mathrm{~W}-12 \mathrm{P} 3$ & 135 & 405 & 33 & - & 130 & - & - & B \\
\hline $34 \mathrm{~N} / 2 \mathrm{~W}-13 \mathrm{H} 1$ & 80 & 12 & - & 77 & 50 & - & - & - \\
\hline $34 \mathrm{~N} / 2 \mathrm{~W}-13 \mathrm{H} 2$ & 80 & 180 & 35 & 70 & 170 & - & - & B \\
\hline $34 \mathrm{~N} / 2 \mathrm{~W}-24 \mathrm{~K} 1$ & 60 & 203 & 20 & - & 85 & - & 77 & M \\
\hline $34 \mathrm{~N} / 2 \mathrm{~W}-24 \mathrm{~L} 2$ & 60 & 238 & 45 & - & 160 & - & - & $\mathrm{B}$ \\
\hline $35 \mathrm{~N} / 1 \mathrm{~W}-07 \mathrm{~N} 1$ & 130 & 128 & 118 & 18 & 78 & - & - & G \\
\hline 35N/1W-31D1 & 75 & 305 & 30 & 60 & 140 & - & - & B \\
\hline 35N/1W-31M1 & 170 & 245 & 20 & - & 74 & - & - & $\mathrm{B}$ \\
\hline $35 \mathrm{~N} / 2 \mathrm{~W}-01 \mathrm{M} 2$ & 60 & 80 & 60 & 15 & 360 & - & - & G \\
\hline $35 \mathrm{~N} / 2 \mathrm{~W}-01 \mathrm{~N} 3$ & 40 & 64 & 58 & 8 & 110 & - & - & G \\
\hline $35 \mathrm{~N} / 2 \mathrm{~W}-01 \mathrm{P} 3$ & 40 & 50 & - & 11 & 300 & - & - & - \\
\hline $35 \mathrm{~N} / 2 \mathrm{~W}-02 \mathrm{P} 1$ & 40 & 60 & 55 & - & 100 & 50 & - & $\mathrm{G}$ \\
\hline $35 \mathrm{~N} / 2 \mathrm{~W}-02 \mathrm{P} 2$ & 70 & 99 & 94 & -8 & 180 & - & - & G \\
\hline $35 \mathrm{~N} / 2 \mathrm{~W}-02 \mathrm{P} 3$ & 70 & 76 & 71 & 5 & 100 & - & - & G \\
\hline $35 \mathrm{~N} / 2 \mathrm{~W}-10 \mathrm{~B} 3$ & 40 & 33 & 28 & 19 & 30 & - & - & G \\
\hline $35 \mathrm{~N} / 2 \mathrm{~W}-10 \mathrm{G} 1$ & 120 & 135 & 130 & -2 & 120 & - & - & G \\
\hline $35 \mathrm{~N} / 2 \mathrm{~W}-10 \mathrm{~J} 2$ & 170 & 180 & 180 & 7 & 100 & - & - & G \\
\hline $35 \mathrm{~N} / 2 \mathrm{~W}-10 \mathrm{~K} 1$ & 140 & 135 & 125 & 19 & 100 & - & - & G \\
\hline $35 \mathrm{~N} / 2 \mathrm{~W}-10 \mathrm{Q} 4$ & 150 & 153 & 153 & 17 & 130 & - & - & G \\
\hline $35 \mathrm{~N} / 2 \mathrm{~W}-10 \mathrm{Q} 5$ & 160 & 150 & 151 & - & 150 & - & - & G \\
\hline $35 \mathrm{~N} / 2 \mathrm{~W}-11 \mathrm{~A} 1$ & 60 & 104 & 98 & 12 & 110 & - & - & G \\
\hline $35 \mathrm{~N} / 2 \mathrm{~W}-11 \mathrm{~B} 1$ & 60 & 130 & - & -60 & 54 & - & - & - \\
\hline $35 \mathrm{~N} / 2 \mathrm{~W}-11 \mathrm{C} 2$ & 90 & 102 & 97 & 8 & 30 & 34 & - & G \\
\hline $35 \mathrm{~N} / 2 \mathrm{~W}-11 \mathrm{D} 1$ & 60 & 68 & 63 & - & 32 & 39 & 34 & G \\
\hline $35 \mathrm{~N} / 2 \mathrm{~W}-11 \mathrm{~F} 1$ & 130 & 143 & 138 & - & 34 & - & - & $\mathrm{G}$ \\
\hline $35 \mathrm{~N} / 2 \mathrm{~W}-11 \mathrm{~J} 1$ & 170 & 185 & 185 & 4 & 34 & 45 & - & G \\
\hline $35 \mathrm{~N} / 2 \mathrm{~W}-11 \mathrm{~K} 1$ & 145 & 160 & 160 & 0 & 80 & - & - & G \\
\hline $35 \mathrm{~N} / 2 \mathrm{~W}-11 \mathrm{~N} 1$ & 130 & 154 & - & - & 38 & - & - & - \\
\hline $35 \mathrm{~N} / 2 \mathrm{~W}-12 \mathrm{~B} 3$ & 60 & 74 & 74 & 8 & 48 & - & - & G \\
\hline $35 \mathrm{~N} / 2 \mathrm{~W}-12 \mathrm{C} 3$ & 130 & 141 & 136 & - & 32 & - & - & G \\
\hline $35 \mathrm{~N} / 2 \mathrm{~W}-12 \mathrm{D} 2$ & 90 & 95 & 90 & 8 & 30 & 44 & - & G \\
\hline $35 \mathrm{~N} / 2 \mathrm{~W}-12 \mathrm{D} 3$ & 130 & 158 & 152 & - & 28 & - & - & G \\
\hline $35 \mathrm{~N} / 2 \mathrm{~W}-12 \mathrm{E} 2$ & 220 & 243 & 243 & 12 & 32 & - & - & G \\
\hline $35 \mathrm{~N} / 2 \mathrm{~W}-12 \mathrm{~F} 2$ & 22 & 29 & - & 3 & 140 & 90 & - & - \\
\hline $35 \mathrm{~N} / 2 \mathrm{~W}-12 \mathrm{~L} 2$ & 60 & 70 & 70 & 4 & 80 & 57 & 56 & G \\
\hline $35 \mathrm{~N} / 2 \mathrm{~W}-12 \mathrm{M} 2$ & 165 & 186 & 186 & -2 & 80 & - & - & G \\
\hline $35 \mathrm{~N} / 2 \mathrm{~W}-12 \mathrm{P} 1$ & 18 & 40 & 34 & 1 & 110 & - & - & G \\
\hline $35 \mathrm{~N} / 2 \mathrm{~W}-12 \mathrm{Q} 1$ & 60 & 80 & 80 & 6 & 200 & - & - & G \\
\hline $35 \mathrm{~N} / 2 \mathrm{~W}-12 \mathrm{Q} 2$ & 60 & 66 & 66 & 18 & 100 & - & - & G \\
\hline $35 \mathrm{~N} / 2 \mathrm{~W}-12 \mathrm{R} 1$ & 165 & 181 & 180 & -3 & 190 & - & - & G \\
\hline $35 \mathrm{~N} / 2 \mathrm{~W}-13 \mathrm{~B} 1$ & 100 & 116 & 116 & 2 & 74 & - & - & G \\
\hline $35 \mathrm{~N} / 2 \mathrm{~W}-13 \mathrm{D} 1$ & 115 & 125 & 125 & 6 & 78 & - & - & G \\
\hline
\end{tabular}


Table 1. Summary of concentrations of chlorides, physical data, and hydrologic data for wells and a spring sampled in 1997 on Lopez Island-Continued

\begin{tabular}{|c|c|c|c|c|c|c|c|c|}
\hline \multirow{2}{*}{$\begin{array}{l}\text { Station name (town- } \\
\text { ship/range-section and } \\
\text { sequence number) }\end{array}$} & \multirow{2}{*}{$\begin{array}{l}\text { Altitude of land } \\
\text { surface (feet) }\end{array}$} & \multirow{2}{*}{$\begin{array}{l}\text { Well depth } \\
\text { (feet below } \\
\text { land surface) }\end{array}$} & \multirow{2}{*}{$\begin{array}{l}\text { Depth to first } \\
\text { opening of } \\
\text { well (feet) }\end{array}$} & \multirow{2}{*}{$\begin{array}{c}\text { Spring } 1997 \\
\text { water-level } \\
\text { altitude (feet) }\end{array}$} & \multicolumn{3}{|c|}{$\begin{array}{l}\text { Chloride dissolved } \\
\text { (mg/L as CL) }\end{array}$} & \multirow{2}{*}{$\begin{array}{c}\text { Hydrogeologic } \\
\text { unit }\end{array}$} \\
\hline & & & & & $\begin{array}{c}\text { Spring } \\
1997\end{array}$ & $\begin{array}{l}\text { Spring } \\
1981\end{array}$ & $\begin{array}{l}\text { Spring } \\
1978\end{array}$ & \\
\hline $35 \mathrm{~N} / 2 \mathrm{~W}-13 \mathrm{E} 1$ & 100 & 114 & 115 & 3 & 40 & - & - & $\mathrm{G}$ \\
\hline $35 \mathrm{~N} / 2 \mathrm{~W}-13 \mathrm{M} 1$ & 100 & 114 & 114 & 3 & 48 & - & - & G \\
\hline $35 \mathrm{~N} / 2 \mathrm{~W}-13 \mathrm{R} 1$ & 85 & 151 & 146 & 4 & 160 & - & - & G \\
\hline $35 \mathrm{~N} / 2 \mathrm{~W}-14 \mathrm{~A} 2$ & 130 & 150 & 150 & 4 & 48 & - & - & G \\
\hline $35 \mathrm{~N} / 2 \mathrm{~W}-14 \mathrm{~B} 2$ & 135 & 164 & 164 & -0 & 62 & - & - & G \\
\hline $35 \mathrm{~N} / 2 \mathrm{~W}-14 \mathrm{E} 1$ & 100 & 113 & 108 & -0 & 50 & 39 & 27 & G \\
\hline $35 \mathrm{~N} / 2 \mathrm{~W}-14 \mathrm{~F} 1$ & 135 & 142 & 136 & 10 & 32 & - & - & G \\
\hline $35 \mathrm{~N} / 2 \mathrm{~W}-14 \mathrm{~J} 2$ & 100 & 160 & 100 & -2 & 100 & - & - & G \\
\hline $35 \mathrm{~N} / 2 \mathrm{~W}-14 \mathrm{M} 1$ & 110 & 113 & 105 & 10 & 52 & - & - & $\mathrm{G}$ \\
\hline $35 \mathrm{~N} / 2 \mathrm{~W}-14 \mathrm{M} 2$ & 180 & 155 & 136 & 45 & 50 & - & - & G \\
\hline $35 \mathrm{~N} / 2 \mathrm{~W}-14 \mathrm{~N} 1$ & 170 & 137 & 132 & 48 & 120 & - & - & G \\
\hline $35 \mathrm{~N} / 2 \mathrm{~W}-15 \mathrm{~B} 1$ & 130 & 158 & 153 & - & 290 & - & 200 & G \\
\hline $35 \mathrm{~N} / 2 \mathrm{~W}-15 \mathrm{H} 1$ & 70 & 102 & 102 & -2 & 90 & - & - & G \\
\hline $35 \mathrm{~N} / 2 \mathrm{~W}-15 \mathrm{R} 3$ & 20 & 65 & 61 & 0 & 18 & - & - & $\mathrm{G}$ \\
\hline $35 \mathrm{~N} / 2 \mathrm{~W}-21 \mathrm{~J} 1$ & 20 & 302 & 23 & - & 250 & - & - & $\mathrm{B}$ \\
\hline $35 \mathrm{~N} / 2 \mathrm{~W}-22 \mathrm{D} 1$ & 15 & 150 & 10 & - & 86 & 150 & - & B \\
\hline 35N/2W-22J1 & 45 & 50 & 46 & - & 16 & 63 & 73 & B \\
\hline $35 \mathrm{~N} / 2 \mathrm{~W}-22 \mathrm{~L} 1$ & 18 & 345 & 20 & -9 & 94 & - & - & B \\
\hline $35 \mathrm{~N} / 2 \mathrm{~W}-23 \mathrm{C} 1$ & 80 & 100 & 99 & 0 & 32 & - & - & G \\
\hline $35 \mathrm{~N} / 2 \mathrm{~W}-23 \mathrm{D} 1$ & 80 & 99 & 89 & - & 46 & - & - & G \\
\hline $35 \mathrm{~N} / 2 \mathrm{~W}-23 \mathrm{G} 1$ & 160 & 173 & 173 & 3 & 14 & - & - & B \\
\hline $35 \mathrm{~N} / 2 \mathrm{~W}-23 \mathrm{G} 2$ & 182 & 216 & 216 & -12 & 30 & - & - & B \\
\hline $35 \mathrm{~N} / 2 \mathrm{~W}-23 \mathrm{~J} 2$ & 220 & 229 & 211 & 12 & 30 & - & - & G \\
\hline $35 \mathrm{~N} / 2 \mathrm{~W}-23 \mathrm{~K} 2$ & 205 & 211 & 211 & 5 & 30 & - & - & G \\
\hline $35 \mathrm{~N} / 2 \mathrm{~W}-24 \mathrm{~A} 2$ & 98 & 265 & 106 & 33 & 150 & - & - & G \\
\hline $35 \mathrm{~N} / 2 \mathrm{~W}-24 \mathrm{E} 1$ & 100 & 120 & 112 & - & 110 & - & - & G \\
\hline $35 \mathrm{~N} / 2 \mathrm{~W}-24 \mathrm{H} 1$ & 120 & 100 & - & 110 & 130 & 92 & - & - \\
\hline $35 \mathrm{~N} / 2 \mathrm{~W}-24 \mathrm{~K} 1$ & 80 & 90 & 85 & 35 & 100 & - & - & G \\
\hline $35 \mathrm{~N} / 2 \mathrm{~W}-24 \mathrm{P} 1$ & 250 & 302 & 302 & 67 & 100 & - & - & G \\
\hline $35 \mathrm{~N} / 2 \mathrm{~W}-24 \mathrm{Q} 1$ & 44 & 91 & 86 & 26 & 100 & - & - & G \\
\hline $35 \mathrm{~N} / 2 \mathrm{~W}-25 \mathrm{~B} 1$ & 40 & 141 & 95 & - & 32 & 39 & 74 & G \\
\hline $35 \mathrm{~N} / 2 \mathrm{~W}-25 \mathrm{~F} 1$ & 139 & 237 & 83 & - & 40 & - & - & B \\
\hline $35 \mathrm{~N} / 2 \mathrm{~W}-25 \mathrm{P} 1$ & 190 & 300 & 40 & 50 & 60 & - & - & B \\
\hline $35 \mathrm{~N} / 2 \mathrm{~W}-25 \mathrm{Q} 1$ & 125 & 60 & 60 & 84 & 82 & 64 & - & G \\
\hline $35 \mathrm{~N} / 2 \mathrm{~W}-25 \mathrm{R} 1$ & 60 & 215 & 26 & 49 & 94 & 87 & - & B \\
\hline $35 \mathrm{~N} / 2 \mathrm{~W}-25 \mathrm{R} 2$ & 60 & 382 & 318 & - & 220 & - & - & B \\
\hline $35 \mathrm{~N} / 2 \mathrm{~W}-26 \mathrm{~B} 1$ & 218 & 220 & 220 & 9 & 64 & - & - & G \\
\hline $35 \mathrm{~N} / 2 \mathrm{~W}-26 \mathrm{D} 1$ & 290 & 322 & 296 & 10 & 40 & - & - & G \\
\hline $35 \mathrm{~N} / 2 \mathrm{~W}-26 \mathrm{G} 1$ & 265 & 280 & 275 & 10 & 20 & - & - & G \\
\hline $35 \mathrm{~N} / 2 \mathrm{~W}-26 \mathrm{~K} 1$ & 230 & 261 & 261 & 1 & 40 & 38 & - & G \\
\hline $35 \mathrm{~N} / 2 \mathrm{~W}-26 \mathrm{M} 1$ & 220 & 249 & 241 & - & 30 & 25 & - & G \\
\hline $35 \mathrm{~N} / 2 \mathrm{~W}-26 \mathrm{P} 1$ & 190 & 426 & 426 & -7 & 160 & - & - & G \\
\hline $35 \mathrm{~N} / 2 \mathrm{~W}-27 \mathrm{E} 1$ & 33 & 38 & 38 & 5 & 30 & - & - & G \\
\hline $35 \mathrm{~N} / 2 \mathrm{~W}-27 \mathrm{E} 2$ & 22 & 22 & 18 & 13 & 12 & - & - & G \\
\hline $35 \mathrm{~N} / 2 \mathrm{~W}-27 \mathrm{~F} 2$ & 70 & 78 & 78 & 12 & 40 & - & - & G \\
\hline $35 \mathrm{~N} / 2 \mathrm{~W}-27 \mathrm{~F} 3$ & 40 & - & - & 9 & 30 & - & - & - \\
\hline $35 \mathrm{~N} / 2 \mathrm{~W}-27 \mathrm{~J} 1$ & 230 & 241 & 241 & 102 & 28 & - & - & G \\
\hline $35 \mathrm{~N} / 2 \mathrm{~W}-27 \mathrm{~J} 2$ & 250 & 270 & 165 & -5 & 20 & - & - & G \\
\hline $35 \mathrm{~N} / 2 \mathrm{~W}-28 \mathrm{~K} 3$ & 110 & 122 & 117 & 8 & 150 & - & - & G \\
\hline $35 \mathrm{~N} / 2 \mathrm{~W}-28 \mathrm{Q} 1$ & 190 & 212 & 207 & -3 & 60 & 72 & - & G \\
\hline $35 \mathrm{~N} / 2 \mathrm{~W}-28 \mathrm{R} 1$ & 150 & 155 & 155 & 11 & 60 & 55 & - & G \\
\hline $35 \mathrm{~N} / 2 \mathrm{~W}-33 \mathrm{G} 1$ & 130 & 140 & 140 & 15 & 420 & 360 & 390 & G \\
\hline $35 \mathrm{~N} / 2 \mathrm{~W}-33 \mathrm{~J} 2$ & 190 & 220 & - & -6 & 150 & - & - & - \\
\hline $35 \mathrm{~N} / 2 \mathrm{~W}-33 \mathrm{R} 2$ & 190 & 190 & 189 & 16 & 24 & - & - & G \\
\hline $35 \mathrm{~N} / 2 \mathrm{~W}-34 \mathrm{~F} 1$ & 180 & 194 & 188 & -1 & 68 & 75 & - & G \\
\hline $35 \mathrm{~N} / 2 \mathrm{~W}-34 \mathrm{~K} 1$ & 190 & 203 & 203 & 4 & 100 & - & - & G \\
\hline $35 \mathrm{~N} / 2 \mathrm{~W}-35 \mathrm{D} 1$ & 205 & 191 & 191 & 22 & 28 & - & - & G \\
\hline $35 \mathrm{~N} / 2 \mathrm{~W}-35 \mathrm{H} 1$ & 280 & 255 & 30 & 270 & 28 & 38 & - & B \\
\hline $35 \mathrm{~N} / 2 \mathrm{~W}-35 \mathrm{~L} 1$ & 210 & 310 & 131 & - & 28 & 23 & - & B \\
\hline $35 \mathrm{~N} / 2 \mathrm{~W}-35 \mathrm{M} 1$ & 185 & 219 & 219 & 3 & 100 & - & - & G \\
\hline $35 \mathrm{~N} / 2 \mathrm{~W}-36 \mathrm{D} 1$ & 290 & 485 & 300 & - & 50 & 51 & - & M \\
\hline $36 \mathrm{~N} / 2 \mathrm{~W}-36 \mathrm{~N} 1$ & 80 & 149 & 20 & - & 24 & - & - & B \\
\hline
\end{tabular}




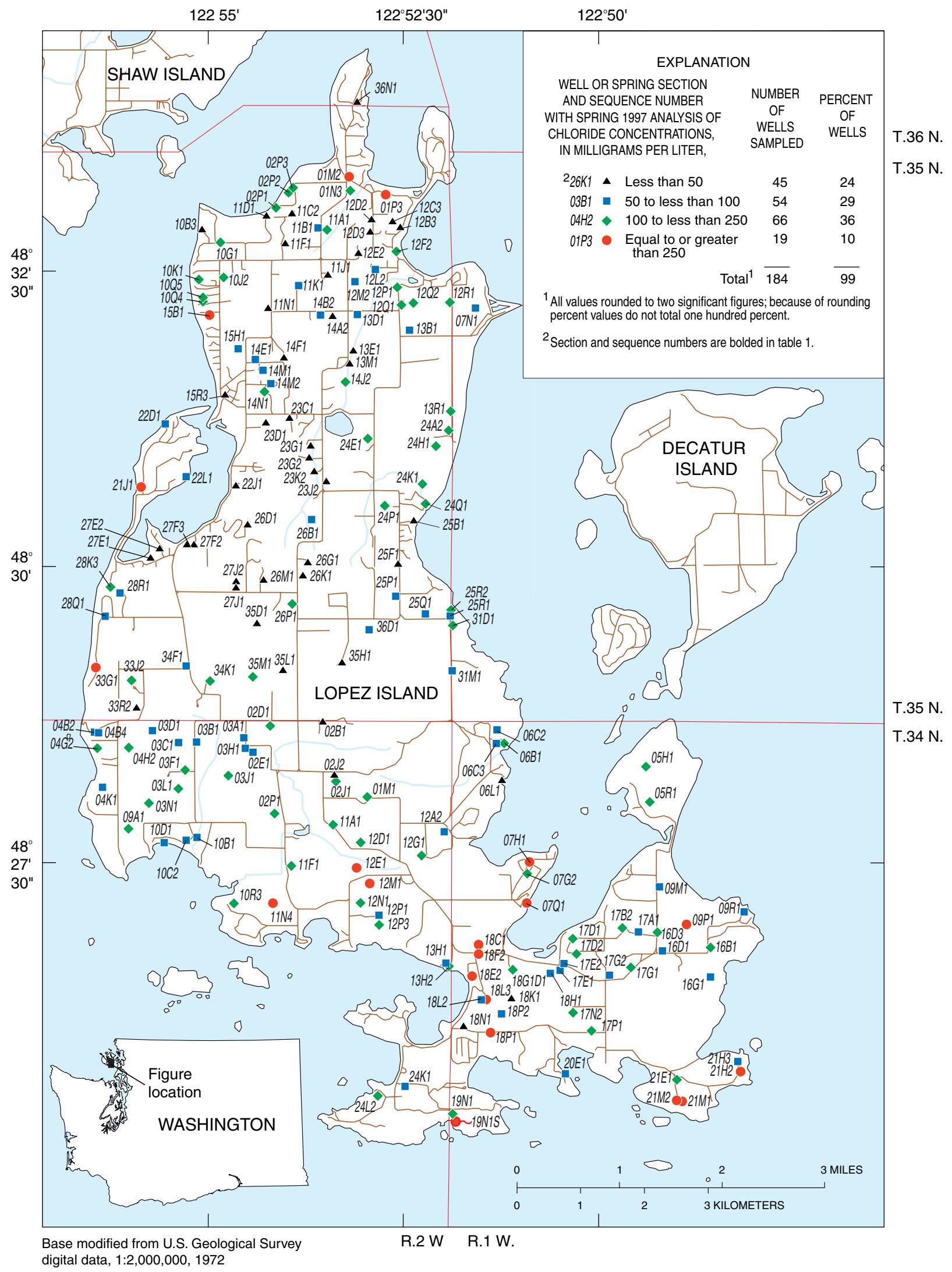

Figure 3. Areal distribution of chloride concentrations from wells or spring on Lopez Island measured in the spring of 1997. 
Table 2. Chloride concentrations in ground-water samples collected April 1981 and in late April to early June 1997, Lopez Island, Washington

[Chloride data rounded to two significant digits]

\begin{tabular}{|c|c|c|c|c|c|c|c|c|}
\hline \multirow{2}{*}{$\begin{array}{l}\text { Group of } \\
\text { samples }\end{array}$} & \multirow[b]{2}{*}{ Year } & \multirow{2}{*}{$\begin{array}{l}\text { Number of } \\
\text { samples }\end{array}$} & \multicolumn{5}{|c|}{ Chloride concentration, in milligrams per liter } & \multirow[b]{2}{*}{ P-value } \\
\hline & & & Minimum & $\begin{array}{c}\text { 25th } \\
\text { percentile }\end{array}$ & Median & $\begin{array}{c}\text { 75th } \\
\text { percentile }\end{array}$ & Maximum & \\
\hline \multirow{3}{*}{ Paired, all } & 1981 & 44 & 23 & 45 & 67 & 100 & 360 & \multirow{3}{*}{${ }^{1} 0.01$} \\
\hline & & & & & & & & \\
\hline & 1997 & 44 & 16 & 42 & 86 & 140 & 420 & \\
\hline \multirow{2}{*}{$\begin{array}{l}\text { Paired, } \\
\text { within } 1,500 \\
\text { feet of } \\
\text { shoreline }\end{array}$} & 1981 & 28 & 34 & 54 & 86 & 140 & 360 & \multirow[b]{2}{*}{${ }^{1} 0.06$} \\
\hline & 1997 & 28 & 16 & 59 & 90 & 160 & 420 & \\
\hline
\end{tabular}

${ }^{1}$ A Wilcoxon signed-rank test (one-sided) was used to test the hypothesis that the chloride concen-trations in 1997 were not greater than chloride concentrations in 1981. The test was conducted using onlywells that were sampled in 1981 and again in 1997. P-values less than 0.05 indicate a significant increase in chloride concentrations from 1981 to 1997.

When seawater intrudes, three trends are usually apparent. First, chloride concentrations at a given site may increase over time. Second, for wells open at the same depth, there may be a strong relation between chloride concentrations and a well's distance from the shoreline, with chlorides being greater the closer a well is to shore. Third, chloride concentrations at a given site may increase with depth.

The first trend was found on Lopez Island. Chloride samples collected from the same wells in 1981 and in 1997 showed a statistically significant increase in concentration over time (table 2). But no trends were found between chloride concentrations and distance from shore or between chloride concentrations and the depth of a well's open interval. These trends may not have been apparent because of wells too shallow to be strongly influenced by the freshwater-saltwater transition zone or because of the effects of sea spray, varying lithologies, different ground-water levels, possible pumping before sampling, or uneven areal distribution of sampled sites.

The 1981 and 1997 chloride data were subjected to two statistical tests: Wilcoxon signed-rank tests on (1) all paired samples and (2) paired samples from near-shoreline wells within 1,500 feet of the shoreline (Helsel and Hirsch, 1992). Using paired samples removes the influence of many environmental factors, so the test more accurately indicates real differences in chloride concentrations over time. The paired samples from nearshoreline wells were tested because one may expect the wells closer to the shoreline to be more sensitive to seawater intrusion. The wells tested for chlorides in 1981 and 1997 showed a statistically significant increase in concentration. But no significant increase in concentration was found for the near-shoreline wells (table 2).

Chloride concentrations in excess of 100 $\mathrm{mg} / \mathrm{L}$ suggested seawater intrusion, and the statistical tests indicated that concentrations had increased over time. But the data did not show trends of consistently higher concentrations near the shoreline or consistent increases of concentration with depth. Thus, further investigations are needed to rule out sources of chloride other than seawater intrusion.

\section{HOW CHLORIDE AFFECTS THE QUALITY OF THE WATER?}

According to the U.S. Environmental Protection Agency (EPA), water with high chloride content may, among other things, cause high blood pressure; taste salty; corrode pipes, fixtures, and appliances; and blacken and pit stainless steel. The EPA has set a Secondary Maximum Contaminant Level (SMCL) of $250 \mathrm{mg} / \mathrm{L}$ for chlorides. An SMCL is the concentration limit for a nuisance contaminant that could affect the aesthetic quality of water by causing taste, odor, or staining problems (U.S. Environmental Protection Agency, 1996). 


\section{FUTURE STUDIES}

Future studies like these examples could help assist understanding of seawater intrusion on Lopez Island:

- Twice-a-year sampling of a network of monitoring wells for specific conductance and chloride concentrations to observe minimum values in early spring and maximum values in late summer or early fall.

- Detailed evaluation of the hydrogeologic conditions that control the movement of the freshwater-seawater transition zone. A three-dimensional digital model of the ground-water system would enable this evaluation and this would require definition of hydrogeologic boundaries, hydraulic properties, water levels, and a water budget.

- Determination and mapping of the location of seawater versus the location of the freshwater aquifers using geophysical methods or electromagnetic induction.

-Target drilling and installation of multi-depth monitoring wells in suspect nearshore areas; constructing flow path sections.

-Installment of precipitation collectors to measure the amount of chloride in precipitation.

\section{CITED REFERENCES}

Dion, N.P., and Sumioka, S.S., 1984, Seawater intrusion into coastal aquifers in Washington, 1978: U.S. Geological Survey Water-Supply Bulletin 56, 10 of 14 pls.

Freeze, R.A., and Cherry, J.A., 1979, Groundwater: Englewood Cliffs, N.J., PrenticeHall Inc., 604 p.

Friedman, L.C., and Erdmann, D.E., 1982, Quality assurance practices for the chemical and biological analyses of water and fluvial sediments: U.S. Geological Survey Techniques of Water-Resources Investigations, Book 5, ch. A6, 181 p.

Garcia, K.T., Maddy, D.V., Lopp, L.E., Jackson, L.D., Couope, R.H., and Schertz, Terey L., 1997, User's manual for the National Water Information System of the U.S. Geological Survey, chap. 2: U.S. Geological Survey Open-File Report 97-634, unpaginated.

Helsel, D.R., and Hirsch, R.M., 1992, Statistical methods in water resources: New York, Elsevier Science Publishing Company, 522 p.

Jones, M.A., 1985, Occurrence of ground water and potential for seawater intrusion, Island County, Washington: U.S. Geological Survey Water-Resources Investigations Report 85-4046, 6 pls.

Oregon Climate Service, Oregon State University, 1999, 1961-1990 annual average precipitation contours: Washington, accessed June 2, 1999, URL http://www.ocs.orst. edu/pub/maps/Precipitation/Total/States/WA/wa.gif.

U.S. Environmental Protection Agency, 1996, Drinking water regulation and health advisories: U.S. Environmental Protection Agency, Office of Water, EPA 822-R-96001, about 12 p.

Whiteman, K.J., Molenaar, Dee, Bortleson, G.C., and Jacoby, J.M., 1983, Occurrence, quality, and use of ground water in Orcas, San Juan, Lopez and Shaw Islands, San Juan County, Washington: U.S. Geological Survey Water-Resources Investigations Report 83-4019, 1-12 pls.

\section{ACKNOWLEDGMENTS}

The USGS thanks the many well owners and well drillers who supplied well records and other information and allowed access to their wells, and to Dave Garland of Washington State Department of Ecology.

Adapted \& edited by James Lyles, designed by Connie Dean, illustrated by Deanna Walth, and formatted by Ginger Renslow.

\section{FOR MORE INFORMATION CONTACT}

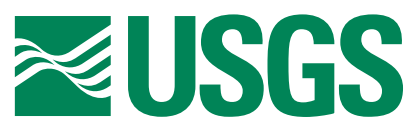

Laura Orr, U.S. Geological Survey1201 Pacific Avenue,

Suite 600, Tacoma, Washington 98402

(253) 428-3600 http://wa.water.usgs.gov/

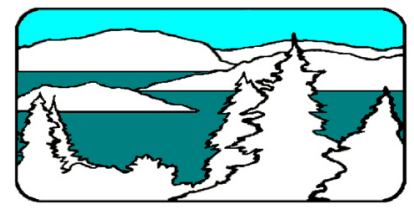

Health \& Community Services San Juan County

P.O. Box 607 - 145 Rhone,

Friday Harbor, WA 98250

Phone (360) 378-4474 Fax (360) 378-7036

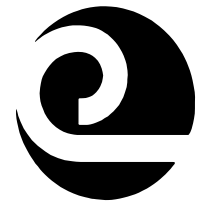

San Juan County Conservation District 350 Court Street \#10, Friday Harbor, Washington 98250 (360) 378-6621 FAX: (360) 378-2445 